\title{
GEOGRAPHY OF SPIN SYMPLECTIC FOUR-MANIFOLDS WITH ABELIAN FUNDAMENTAL GROUP
}

\author{
RAFAEL TORRES \\ (Received 8 May 2010; accepted 18 March 2011) \\ Communicated by M. K. Murray
}

\begin{abstract}
We study the geography and botany of symplectic spin four-manifolds with abelian fundamental group. By building on the constructions of J. Park and of B. D. Park and Szabó, we can give alternative proofs and extend several results on the geography of simply connected four-manifolds to the nonsimply connected realm.
\end{abstract}

2010 Mathematics subject classification: primary 57R55; secondary 57R17.

Keywords and phrases: symplectic geometry, geography problem, torus surgery, exotic smooth structures.

\section{Introduction}

Due to the wild and untamed nature of smooth four-manifolds, efforts towards a classification are quite involved. The addition of a symplectic structure has led to an impressive improvement in our understanding of these objects. The geography problem, first proposed by McCarthy and Wolfson [18], consists of the existence part of a possible classification: given a fundamental group, Euler characteristic and signature, is there a symplectic spin four-manifold with these topological invariants? The (lack of) uniqueness of such manifolds is known as the botany problem: how many diffeomorphism classes are there for the symplectic manifold constructed with the given topological invariants?

The geography and botany of irreducible spin simply connected four-manifolds have been studied in depth [6, 12, 20-22, 24], so that most of the existence questions have been settled. The recent addition of Luttinger surgery $[3,17]$ to the repertoire of symplectic constructions was extremely effective. Not only did it lead to an impressive development in our understanding of simply connected four-manifolds [1, 2, 4], but it also facilitated the study of the geography for other fundamental groups [4, 27, 28]. The purpose of this paper is to extend results on simply connected manifolds to those whose fundamental group is an abelian group of small rank.

(C) 2011 Australian Mathematical Publishing Association Inc. 1446-7887/2011 \$16.00 
The progress on the botany has not been any less impressive. Fintushel and Stern's work on surgery on null-homologous tori $[8,9]$ unveiled a myriad of exotic smooth structures that were previously out of reach through an elegant geometric and topological mechanism. The same authors in joint work with B. D. Park [5] exploited a duality between Luttinger surgery and its counterpart on null-homologous tori that enabled the parallel study of the symplectic geography and its botany used by many authors these days, this note included.

To put the results of this paper into context, we give a rough outline of current knowledge about the geography of symplectic spin four-manifolds with trivial fundamental group $\pi_{1}$. These manifolds have an Euler characteristic $e$ and a holomorphic Euler characteristic $\chi_{h}$. Extending and diagonalizing the intersection form of the manifold, the second Betti number $b_{2}$ may be written as $b_{2}^{+}+b_{2}^{-}$, where $b_{2}^{+}$ and $b_{2}^{-}$are the number of times +1 and -1 appear in the diagonal. The signature $\sigma$ is then $b_{2}^{+}-b_{2}^{-}$; finally, $\chi_{h}=\frac{1}{4}(e+\sigma)$ and $c_{1}^{2}=2 e+3 \sigma$, where $c_{1}^{2}$ is the selfintersection of the first Chern class. B. D. Park and Szabó [22] proved that there is a simply connected spin irreducible symplectic four-manifold with every allowed homeomorphism type such that $0 \leq c_{1}^{2}<8 \chi_{h}$ and $b_{2}^{+}$is odd [22, Theorem 1.1]. J. Park obtained a similar but much broader result [21, Theorem 1.1], which also treated spin symplectic simply connected four-manifolds of zero and positive signature. In particular, he cleverly used a complex spin surface built by Persson et al. [23] to produce an infinite number of exotic smooth structures on $(2 n+1)\left(S^{2} \times S^{2}\right)$, the connected sum of $2 n+1$ copies of $S^{2} \times S^{2}$, for all sufficiently large numbers $n$.

Our first result concerns the geography of spin manifolds with negative signature. It provides an extension of the result of B. D. Park and Szabó to manifolds with nontrivial abelian fundamental groups. In the simply connected case, we also offer an alternative proof to their theorem.

THEOREM 1.1. Let $n$ and $s$ be positive integers, $p$ and $q$ be integers greater than 1 , and $G$ be $\{1\}, \mathbb{Z}_{p}, \mathbb{Z}_{p} \oplus \mathbb{Z}_{q}$ (if $n \geq 2$ ) or $\mathbb{Z}, \mathbb{Z} \oplus \mathbb{Z}_{p}, \mathbb{Z} \oplus \mathbb{Z}$ (if $n \geq 1$ ). If

$$
(c, \chi)=(8 n-8,2 s+n-1),
$$

then there exists an irreducible symplectic spin four-manifold $X$ such that

$$
\pi_{1}(X)=G \quad \text { and } \quad\left(c_{1}^{2}(X), \chi_{h}(X)\right)=(c, \chi) .
$$

For four-manifolds with nonnegative signature, by following closely J. Park's main construction [21], one obtains the following result.

Theorem 1.2. Let $G$ be one of $\{1\}, \mathbb{Z}_{p}, \mathbb{Z}_{p} \oplus \mathbb{Z}_{q}, \mathbb{Z}, \mathbb{Z} \oplus \mathbb{Z}_{p}, \mathbb{Z} \oplus \mathbb{Z}$, where $p$ and $q$ are integers greater than 1 . For all but finitely many pairs of positive integers $(c, \chi)$ in the region $8 \chi \leq c \leq 8.76 \chi$, there exists an irreducible symplectic spin four-manifold $X$ such that

$$
\pi_{1}(X)=G \quad \text { and } \quad\left(c_{1}^{2}(X), \chi_{h}(X)\right)=(c, \chi) .
$$

Concerning their botany, we have the following two results. 
Proposition 1.3. Let $G$ be $\{1\}, \mathbb{Z}_{p}, \mathbb{Z}_{q} \oplus \mathbb{Z}_{q}$ or $\mathbb{Z}$, where $p \geq 2$ and $q$ is an odd prime number, and $(c, \chi)$ be one of the pairs of integers for which manifolds are constructed in Theorems 1.1 and 1.2. Then there exists an infinite family $\left\{X_{n}\right\}$ of homeomorphic, pairwise nondiffeomorphic irreducible smooth nonsymplectic four-manifolds such that $\pi_{1}(X)=G$ and

$$
\left(c_{1}^{2}\left(X_{n}\right), \chi_{h}\left(X_{n}\right)\right)=(c, \chi) .
$$

Following J. Park, for the manifolds with zero signature of Theorem 1.2, we have the following result.

Corollary 1.4. There exists an integer $N$ such that for all $n \geq N$, each of the homeomorphism types given by the manifolds

$$
(2 n+1)\left(S^{2} \times S^{2}\right) \# L_{p}, \quad(2 n+1)\left(S^{2} \times S^{2}\right) \# L_{q, q}, \quad(2 n)\left(S^{2} \times S^{2}\right) \#\left(S^{1} \times S^{3}\right),
$$

where $p \geq 2$ and $q$ is an odd prime number, has infinitely many exotic irreducible smooth structures. In each case, only one of the exotic manifolds is symplectic.

Here $L_{p}$ stands for the manifold obtained by modifying the product $L(p, 1) \times S^{1}$ of a lens space with the circle as follows. Perform surgery on $L(p, 1) \times S^{1}$ along $\{x\} \times \alpha$, where $x \in L(p, 1)$, to kill the loop corresponding to the generator of the infinite cyclic factor so that the fundamental group $\pi_{1}$ of the resulting manifold is $\mathbb{Z}_{p}$, which comes from the fundamental group of the lens space. If we cut out a loop $\{x\} \times \alpha^{q}$ and glue in a disc to kill the corresponding generator instead, then we obtain a four-manifold with fundamental group $\mathbb{Z}_{p} \oplus \mathbb{Z}_{q}$, which is denoted $L_{p, q}$.

The paper is organized as follows. Section 2 provides the reader with a description of the building blocks and the tools that are employed in our constructions. This section also includes the two crucial lemmas for our results. In Section 3, we prove Theorem 1.1 and half of Proposition 1.3. We provide a description of J. Park's construction in Section 4, as well as proofs of Theorem 1.2 and Corollary 1.4, and the remaining part of the proof of Proposition 1.3.

\section{Tools and raw materials}

2.1. Symplectic sums. Gompf [12] introduced the symplectic sum, a beautiful technique to build symplectic four-manifolds that is now essential to our understanding of symplectic four-manifolds. The next result states the properties of this construction that we will use.

Lemma 2.1 (Gompf [12]). Let $X$ and $Y$ be spin symplectic four-manifolds, both of which contain a symplectic surface $\Sigma_{g}$ of genus $g$ and self-intersection 0 . Then the symplectic sum $X \#_{\Sigma_{g}} Y$ is a spin symplectic irreducible manifold, and

$$
\left(c_{1}^{2}\left(X \#_{\Sigma_{g}} Y\right), \chi_{h}\left(X \#_{\Sigma_{g}} Y\right)\right)=\left(c_{1}^{2}(X)+c_{1}^{2}(Y)+8(g-1), \chi_{h}(X)+\chi_{h}(Y)+(g-1)\right) .
$$


A spin symplectic simply connected four-manifold is irreducible, since its SeibergWitten invariant is nontrivial $[25,26]$. The claim of irreducibility for four-manifolds with abelian fundamental group of small rank constructed in this paper follows from Hamilton and Kotschick [16].

2.2. Luttinger surgery and torus surgery. Carving a torus out of a four-manifold and then gluing it back in differently is a standard topological procedure to produce exotic smooth structures. Recently, this idea has been exploited successfully in three directions. First, perform this operation symplectically by adding Luttinger surgery to the palette of constructions of symplectic manifolds; second, use it to construct not only simply connected symplectic manifolds, but also manifolds with several fundamental groups; and last but not least, use a null-homologous torus that canonically comes out of this surgery, as an effective tool to change the smooth structure at will. We proceed to give an overview of the machinery. For specific details on the construction, the reader is directed to the references given below.

Let $T$ be a torus in $X$ of self-intersection zero, which has a tubular neighborhood $N_{T}$, identified with $T^{2} \times D^{2}$. Let $\alpha$ and $\beta$ be the generators of $\pi_{1}(T)$ and consider the meridian $\mu_{T}$ of $T$ in $X$ and the push-offs $S_{\alpha}^{1}$ and $S_{\beta}^{1}$ in $\partial N_{T}$, identified with $T^{3}$; these are loops homologous in $N_{T}$ to $\alpha$ and $\beta$. The manifold obtained from $X$ by performing a $q / p$ surgery on $T$ along $\beta$ is denoted by

$$
X_{T, \beta}(q / p)=\left(X-N_{T}\right) \cup_{\phi}\left(T^{2} \times D^{2}\right),
$$

where the gluing map $\phi: T^{2} \times \partial D^{2} \rightarrow \partial\left(X-N_{T}\right)$ satisfies $\phi_{*}\left(\left[\partial D^{2}\right]\right)=p\left[S_{\beta}^{1}\right]+q\left[\mu_{T}\right]$ in $H_{1}\left(\partial\left(X-N_{T}\right) ; \mathbb{Z}\right)$. Denote the core torus $S^{1} \times S^{1} \times\{0\}$ in $X_{T, \beta}(q / p)$ by $T_{q / p}$. The surgery reduces $b_{1}$ by one and $b_{2}$ by two.

If $X$ is symplectic and $T$ is Lagrangian, then performing a $1 / p$ surgery on the preferred Lagrangian framing of $N_{T}$ results in $X_{T, \beta}(1 / p)$ being symplectic. This procedure is known as $1 / p$ Luttinger surgery [3]. Concerning the botany, Fintushel et al. [5] introduced a procedure that uses the null-homologous torus $T_{q / p}$ to manufacture infinitely many exotic smooth structures starting with a manifold with nontrivial Seiberg-Witten invariant (for example, the symplectic manifold from which $T_{q / p}$ is obtained), by applying a more general $n / 1$ surgery on $T_{q / p}$ (see [5] or the discussion following [4, Theorem 13] for more details). This manufactures an infinite family $\left\{X_{n}\right\}$ of pairwise nondiffeomorphic nonsymplectic four-manifolds (see Remark 2.5 below).

If $X$ is assumed to be spin, one can endow $X_{T, \beta}(q / p)$ with a spin structure by choosing a suitable bundle automorphism from $T^{2} \times D^{2}$ to $T^{2} \times D^{2}$ as follows. Fix a spin structure on $X-N_{T}$ and one on $T^{2} \times D^{2}$. Their difference is given by an element in $H^{1}\left(T^{2} \times D^{2} ; \mathbb{Z}_{2}\right)$, which may be identified with $H^{1}\left(T^{2} ; \mathbb{Z}_{2}\right)$. On the other hand, this element can readily be seen to be the image of an appropriate bundle automorphism under the coefficient homomorphism from $H^{1}\left(T^{2} ; \mathbb{Z}\right)$ to $H^{1}\left(T^{2} ; \mathbb{Z}_{2}\right)$. Thus, identifying two spin structures on $T^{2} \times D^{2}$, coming from $X-N_{T}$ and from $T^{2} \times D^{2}$, yields a spin structure for $X_{T, \beta}(q / p)$ itself. 
In the rest of this section, we introduce the building blocks in our constructions.

2.3. Surgery on $\boldsymbol{T}^{4}$. This building block will allow us to manipulate the fundamental group of our constructions without changing the Euler characteristic nor the signature. Let $\pi_{1}\left(T^{4}\right)$ be generated by $x, y, a, b$. Removing a surface from a four-manifold normally introduces more generators to the fundamental group of the complement. Baldridge and Kirk [4] showed that the fundamental group of the complement of two Lagrangian tori $T_{1}$ and $T_{2}$ inside the four-torus is generated by four elements, just like the group $\pi_{1}\left(T^{4}\right)$ itself.

Proposition 2.2 (Baldridge and Kirk [4]). The fundamental group of $T^{4}-\left(T_{1} \cup T_{2}\right)$ is generated by the loops $x, y, a, b$ and the relations $[x, a]=[y, a]=1$. The meridians of the tori and the two Lagrangian push-offs of their generators are given by the following formulae:

$$
\begin{array}{lll}
\mu_{1}=\left[b^{-1}, y^{-1}\right], & m_{1}=x, & l_{1}=a, \\
\mu_{2}=\left[x^{-1}, b\right], & m_{2}=y, & l_{2}=b a b^{-1} .
\end{array}
$$

As a corollary, we obtain the following lemma.

LEMMA 2.3. Let $X$ be a simply connected spin symplectic four-manifold containing a symplectic torus $T$ such that $\pi_{1}(X-T)=\{1\}$. There exists a spin symplectic fourmanifold $Z$ with Chern numbers $\chi_{h}(Z)=\chi_{h}(X)$ and $c_{1}^{2}(Z)=c_{1}^{2}(X)$. The fundamental group $\pi_{1}$ of $Z$ can be chosen to be

$$
\mathbb{Z} \oplus \mathbb{Z}, \quad \mathbb{Z} \oplus \mathbb{Z}_{q}, \quad \mathbb{Z},
$$

where $q$ is an integer greater than 1.

Proof. Let the torus $T_{1} \subset T^{4}$ be as in Proposition 2.2. Perturb the symplectic form on $T^{4}$ so that $T_{1}$ becomes symplectic while $T_{2}$ stays Lagrangian (see [12, Lemma 1.6]). The torus $T_{1}$ carries the generators $x$ and $b$. Take the symplectic sum $Y:=T^{4} \#_{T_{1}=T} X$ of $T^{4}$ and $X$ along $T_{1}$ and $T$. Since the meridian of $T$ in $X-T$ is trivial, the relation $[y, b]=1$ holds in the fundamental group of this newly constructed manifold. Therefore the symplectic sum gives rise to a manifold $Y$ for which $\pi_{1}(Y)=\mathbb{Z} \oplus \mathbb{Z}$, with generators $y$ and $b$. We can now apply a $1 / q$ Luttinger surgery to $T_{2}$ to produce a manifold with fundamental group $\mathbb{Z}_{q} \oplus \mathbb{Z}$, where $b$ generates $\mathbb{Z}$.

2.4. Symplectic manifolds with the cohomology of $(2 n-3)\left(S^{2} \times S^{2}\right)$. Fintushel et al. [5] built an infinite family of irreducible pairwise nondiffeomorphic spin fourmanifolds with the same integer cohomology ring as $S^{2} \times S^{2}$. Akhmedov and B. D. Park [2] extended the construction, producing an infinite family of irreducible pairwise nondiffeomorphic spin four-manifolds $\left\{Y_{n}(m) \mid m=1,2,3, \ldots\right\}$ with only one symplectic member with the same integer cohomology ring as $(2 n-3)\left(S^{2} \times S^{2}\right)$ where $n \geq 2$. The characteristic numbers of these manifolds are $e=4 n-4$ and $\sigma=0$; equivalently, $c_{1}^{2}=8 n-8$ and $\chi_{h}=n-1$. 
These manifolds are constructed by applying Luttinger surgery $2 n+3$ times and torus surgery once to $\Sigma_{2} \times \Sigma_{n}$ (the product of a genus 2 surface with a genus $n$ surface). Let $a_{i}$ and $b_{i}$ (where $i=1,2$ ) be the standard generators of $\pi_{1}\left(\Sigma_{2}\right)$ and $c_{j}$ and $d_{j}$ (where $j=1, \ldots, n)$ be the standard generators of $\pi_{1}\left(\Sigma_{n}\right)$. The following relations hold in $\pi_{1}\left(Y_{n}(m)\right)$ :

$$
\begin{aligned}
& {\left[b_{1}^{-1}, d_{1}^{-1}\right]=a_{1}, \quad\left[a_{1}^{-1}, d_{1}\right]=b_{1}, \quad\left[b_{2}^{-1}, d_{2}^{-1}\right]=a_{2}, \quad\left[a_{2}^{-1}, d_{2}\right]=b_{2},} \\
& {\left[d_{1}^{-1}, b_{2}^{-1}\right]=c_{1}, \quad\left[c_{1}^{-1}, b_{2}\right]=d_{1}, \quad\left[d_{2}^{-1}, b_{1}^{-1}\right]=c_{2}, \quad\left[c_{2}^{-1}, b_{1}\right]=d_{2},} \\
& {\left[a_{1}, c_{1}\right]=1, \quad\left[a_{1}, c_{2}\right]=1, \quad\left[a_{1}, d_{2}\right]=1, \quad\left[b_{1}, c_{1}\right]=1,} \\
& {\left[a_{2}, c_{1}\right]=1, \quad\left[a_{2}, c_{2}\right]=1, \quad\left[a_{2}, d_{1}\right]=1, \quad\left[b_{2}, c_{2}\right]=1,} \\
& {\left[a_{1}, b_{1}\right]\left[a_{2}, b_{2}\right]=1, \quad\left[c_{1}, d_{1}\right]\left[c_{2}, d_{2}\right]=1,} \\
& {\left[a_{1}^{-1}, d_{3}^{-1}\right]=c_{3}, \quad\left[a_{2}^{-1}, c_{3}^{-1}\right]=d_{3}, \quad \ldots, \quad\left[a_{1}^{-1}, d_{n}^{-1}\right]=c_{n}, \quad\left[a_{2}^{-1}, c_{n}^{-1}\right]=d_{n},} \\
& {\left[b_{1}, c_{3}\right]=1, \quad\left[b_{2}, d_{3}\right]=1, \quad \ldots, \quad\left[b_{1}, c_{n}\right]=1, \quad\left[b_{2}, d_{n}\right]=1,}
\end{aligned}
$$

and

$$
\prod_{j=2}^{n}\left[c_{j}, d_{j}\right]=1 .
$$

We refer the reader to [2] for further details.

These manifolds are our basic building blocks for manufacturing the fundamental group. We employ them to obtain the following result.

LEMMA 2.4. Let $X$ be a simply connected spin symplectic four-manifold containing a symplectic torus such that $\pi_{1}(X-T)=\{1\}$. Then for all positive integers $n$ there exists a spin symplectic four-manifold $Z$ such that

$$
\left(c_{1}^{2}(Z), \chi_{h}(Z)\right)=\left(c_{1}^{2}(X)+8 n-8, \chi_{h}(X)+n-1\right) .
$$

The fundamental group of $Z$ can be chosen to be
(1) $\mathbb{Z} \oplus \mathbb{Z}$
(4) $\mathbb{Z}_{q}$,
(2) $\mathbb{Z} \oplus \mathbb{Z}_{q}$,
(5) $\mathbb{Z}$,
(3) $\mathbb{Z}_{p} \oplus \mathbb{Z}_{q}$,
(6) $\{1\}$,

where $p$ and $q$ are integers greater than 1 . Furthermore, $Z$ contains a Lagrangian torus such that the fundamental group homomorphism from $\pi_{1}(Z-T)$ to $\pi_{1}(Z)$ induced by inclusion is an isomorphism.

Proof. Consider the case where $n=2$. Let $S$ be the manifold obtained by applying \pm 1 Luttinger surgery five times to $\Sigma_{2} \times \Sigma_{2}$. The operations that are not to be performed are $\left(a_{1}^{\prime} \times c_{1}^{\prime}, a_{1}^{\prime},-1\right),\left(a_{2}^{\prime} \times c_{2}^{\prime}, a_{2}^{\prime},-1\right)$ and $\left(a_{2}^{\prime \prime} \times d_{1}^{\prime}, d_{1}^{\prime},+1\right)$ surgery. Call these three tori $T_{1}, T_{2}$ and $T_{3}$ respectively. In $\pi_{1}(S)$, all the relations from $\pi_{1}\left(Y_{2}(1)\right)$ hold, except for $\left[b_{1}^{-1}, d_{1}^{-1}\right]=a_{1},\left[b_{2}^{-1}, d_{2}^{-1}\right]=a_{2}$ and $\left[c_{2}^{-1}, b_{1}\right]=d_{2}$.

Build the symplectic sum of $X$ and $S$ along the corresponding torus in $X$ and $T_{1}$ in

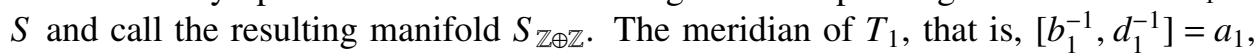


is killed by the symplectic sum construction, and the surviving relations show that the fundamental group $\pi_{1}\left(S_{\mathbb{Z} \oplus \mathbb{Z}}-\left(T_{2} \cup T_{3}\right)\right)$ is generated by the two commuting elements $a_{2}$ and $d_{1}$. The Mayer-Vietoris sequence shows that $H_{1}\left(S_{\mathbb{Z} \oplus \mathbb{Z}}-\left(T_{2} \cup T_{3}\right) ; \mathbb{Z}\right)=\mathbb{Z}^{2}$, and thus $\pi_{1}\left(S_{\mathbb{Z} \oplus \mathbb{Z}}\right)=\mathbb{Z} a_{2} \oplus \mathbb{Z} d_{1}$. It is straightforward to check that $e\left(S_{\mathbb{Z} \oplus \mathbb{Z}}\right)=e(X)+4$ and $\sigma\left(S_{\mathbb{Z} \oplus \mathbb{Z}}\right)=\sigma(X)$.

Note that the geometrical dual torus $T^{\prime}$ of $T_{1}$ is contained in $S_{\mathbb{Z} \oplus \mathbb{Z}}$ and its meridian is trivial in the complement. This implies that $\pi_{1}\left(S_{\mathbb{Z} \oplus \mathbb{Z}}-T^{\prime}\right) \cong \pi_{1}\left(S_{\mathbb{Z} \oplus \mathbb{Z}}\right)=\mathbb{Z}^{2}$. Thus, item (1) of the lemma has been produced.

Applying $\left(a_{2}^{\prime} \times c_{2}^{\prime}, a_{2}^{\prime},-1 / q\right)$, that is, a $-1 / q$ Luttinger surgery to $S_{\mathbb{Z} \oplus \mathbb{Z}}$ on $T_{2}$ along $a_{2}^{\prime}$, produces item (2). Applying $\left(a_{2}^{\prime \prime} \times d_{1}^{\prime}, d_{1}^{\prime},+1 / p\right)$ to the resulting manifold produces item (3) when $p>1$ and item (4) when $p=1$. Applying $\left(a_{2}^{\prime \prime} \times d_{1}^{\prime}, d_{1}^{\prime},+1\right)$ to $S_{\mathbb{Z} \oplus \mathbb{Z}}$ produces item (5), while item (6) is produced by applying both $\left(a_{2}^{\prime \prime} \times d_{1}^{\prime}, d_{1}^{\prime},+1\right)$ and

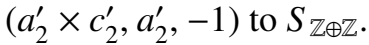

The cases where $n \geq 3$ follow the procedure described above almost verbatim, but substituting $\Sigma_{2} \times \Sigma_{2}$ by $\Sigma_{2} \times \Sigma_{n}$. The details are left to the reader. We point out that the bigger $n$ is, the more Lagrangian tori the resulting manifold contains. For example, the manifold obtained by applying Luttinger surgery to $\Sigma_{2} \times \Sigma_{5}$ contains 12 Lagrangian tori while that obtained from $\Sigma_{2} \times \Sigma_{7}$ has 20 Lagrangian tori; all of these have trivial meridian in homology.

REMARK 2.5. An infinite number of exotic smooth structures can be unveiled at the cost of giving up the symplectic structure. We exemplify the process in the case where the fundamental group is infinite cyclic, while the next paragraph explains why the process works for the other groups. Before applying the last Luttinger surgery to obtain a symplectic manifold with fundamental group $\mathbb{Z}$, one has a symplectic manifold $X_{\mathbb{Z} \oplus \mathbb{Z}}$ for which $\pi_{1}\left(X_{\mathbb{Z} \oplus \mathbb{Z}}\right)=\mathbb{Z} \oplus \mathbb{Z}$. By Taubes' results $[25,26], X_{\mathbb{Z} \oplus \mathbb{Z}}$ has nontrivial SeibergWitten invariants. One can perform a more general torus surgery on $X_{\mathbb{Z} \oplus \mathbb{Z}}$ to obtain a manifold $X_{\mathbb{Z}}$ with fundamental group $\mathbb{Z}$ and nontrivial Seiberg-Witten invariants. The manifold $X_{\mathbb{Z}}$ contains a null-homologous torus $T^{\prime}$. Applying a torus surgery on $T^{\prime}$ yields an infinite family $\left\{X_{n}\right\}$ parametrized by the surgery coefficient $n$. The formula in [19, Theorem 3.4] can be used to prove that the Seiberg-Witten invariants distinguish infinitely many diffeomorphism types within the members of $\left\{X_{n}\right\}$ (see also [5, Corollary 2]).

To conclude our discussion on their homeomorphism type, one must check that these manifolds have the desired fundamental group; we already know that their Chern invariants remain unchanged after the surgery. For this purpose, it suffices to see that the effect of the surgery on the presentation of the fundamental groups is to replace a relation of the form $[a, b]=c^{p}$ by $[a, b]^{n}=c^{p}$ for a given $p$ and $n$ and generators $a, b$. In the proofs of Lemmas 2.3 and 2.4, we argued that the original relation is trivial, and so raising it to any power will result in a trivial relation as well.

2.5. Horikawa surfaces. The complex surfaces satisfying $c_{1}^{2}=2 \chi_{h}-6$ are known as Horikawa surfaces; we denote them throughout this paper by $H\left(8 k^{\prime}-1\right)$. They 
are constructed as branched covers of the Hirzebruch surface $\mathbb{F}_{2 m}$ along disconnected curves. The Chern invariants of $H\left(8 k^{\prime}-1\right)$ are given by $\left(c_{1}^{2}, \chi_{h}\right)=\left(16 k^{\prime}-8,8 k^{\prime}-1\right)$. Moreover, $H\left(8 k^{\prime}-1\right)$ contains an embedded Lagrangian torus which intersects a 2-sphere transversally at one point [6, 24].

2.6. A spin surface of positive signature. Persson et al. [23] constructed a simply connected spin complex surface $Y$ of positive signature which contains a holomorphic curve $\Sigma_{g}$ of genus $g$ and trivial self-intersection. Further, the meridian of this surface in the complement is trivial since $Y$ also contains an embedded 2 -sphere $\mathbb{C P}^{1}$ that intersects $\Sigma_{g}$ transversely at a point. Its Chern invariants are given approximately by $\left(c_{1}^{2}(Y), \chi_{h}(Y)\right) \approx\left(60068 x^{2}, 6857 x^{2}\right)$, where $x$ is a large positive integer that is not computed explicitly.

2.7. Knot surgery on elliptic minimal surfaces. Our last building block is also a classical element in the construction of four-manifolds and we remind the reader of its relevant properties only. Let $E(2 s)$ denote the underlying smooth four-manifold of the simply connected minimal elliptic surface without multiple fibers and with geometric genus $p_{g}=2 s-1$ (see [11] and [13, Proposition 3.1.11]). Its Chern numbers are given by $\left(c_{1}^{2}, \chi_{h}\right)=(0,2 s)$. Notice that in particular $E(2)$ is a $K 3$ surface. In Sections 3 and 4 , it is easy to see where the manifold $E(2 s)$ can be replaced by an exotic version $E(2 s)_{K}$ obtained by knot surgery [8].

\section{Negative signature}

3.1. Examples where $\sigma=\mathbf{- 1 6 s}$ and $s>\mathbf{0}$. We now extend the results of B. D. Park and Szabó [22] to manifolds with abelian fundamental group of small rank.

Proposition 3.1. Let $n$ and $s$ be positive integers, $p$ and $q$ be integers greater than 1 , and $G$ be $\{1\}, \mathbb{Z}_{p}, \mathbb{Z}_{p} \oplus \mathbb{Z}_{q}\left(\right.$ if $n \geq 2$ ) or $\mathbb{Z}, \mathbb{Z} \oplus \mathbb{Z}_{p}$ and $\mathbb{Z} \oplus \mathbb{Z}$ (if $n \geq 1$ ). Then there exists a spin irreducible symplectic manifold $X$ such that

$$
\pi_{1}(X)=G \quad \text { and } \quad\left(c_{1}^{2}(X), \chi_{h}(X)\right)=(8 n-8, n+2 s-1) .
$$

Proof. The proposition follows by employing the symplectic manifold $X=E(2 s)_{K}$ in Lemmas 2.3 and 2.4.

By applying appropriate homeomorphism criteria, we conclude that when $p$ and $q$ are odd prime numbers, the manifolds constructed in Proposition 3.1 for the various choices of $G$ are homeomorphic to the following topological prototypes:

$$
\begin{array}{ll}
G=\{1\} & E(2 s) \#(2 n-2)\left(S^{2} \times S^{2}\right), \\
G=\mathbb{Z}_{p} & E(2 s) \#(2 n-2)\left(S^{2} \times S^{2}\right) \# L_{p}, \\
G=\mathbb{Z}_{q} \oplus \mathbb{Z}_{q} & E(2 s) \#(2 n-2)\left(S^{2} \times S^{2}\right) \# L_{q, q}, \\
G=\mathbb{Z} & E(2 s) \#(2 n-1)\left(S^{2} \times S^{2}\right) \#\left(S^{3} \times S^{1}\right) .
\end{array}
$$

Indeed, the Euler characteristic, the spin property and the signature of the symplectic sum are computed by Lemma 2.1, and torus surgery does not change any 
of these topological invariants. In the simply connected case, the ground-breaking result of Freedman [10] gives the homeomorphism type of the manifolds constructed. Other (less well known but still very significant) results allow us to determine the homeomorphism types for manifolds with nontrivial fundamental group by using the same topological invariants. The criteria of Hambleton and Teichner [15] determines the homeomorphism type for manifolds with infinite cyclic fundamental group. The finite fundamental group cases, both cyclic and noncyclic but abelian of odd order, follow from the criteria of Hambleton and Kreck [14]; one must check that the manifolds constructed share the same $\omega_{2}$-type: this requires verification that the universal covers of the manifolds are spin as well. The hypothesis requiring $q$ to be a prime number in Proposition 1.3 is needed to apply these criteria.

Thus, in light of Remark 2.5, we have the following result.

Corollary 3.2. Each of the manifolds

$$
\begin{array}{ll}
E(2 s) \#(2 n-2)\left(S^{2} \times S^{2}\right), & E(2 s) \#(2 n-2)\left(S^{2} \times S^{2}\right) \# L_{p}, \\
E(2 s) \#(2 n-2)\left(S^{2} \times S^{2}\right) \# L_{q, q}, & E(2 s) \#(2 n-1)\left(S^{2} \times S^{2}\right) \#\left(S^{3} \times S^{1}\right),
\end{array}
$$

where $p$ is an integer greater than 1 and $q$ is an odd prime number, admits infinitely many exotic irreducible smooth structures. In each case, only one of these exotic manifolds is symplectic.

These methods improve the main theorem of Fintushel and Stern [7], who constructed a manifold $X$ homeomorphic to $K 3 \#\left(S^{2} \times S^{2}\right) \#\left(S^{3} \times S^{1}\right)$. We remind the reader that in the noncyclic abelian case, the fundamental group is assumed to be $\pi_{1}=\mathbb{Z}_{q} \oplus \mathbb{Z}_{q}$, where $q$ is a prime number.

3.2. Examples with $\sigma=\mathbf{- 4 8} \boldsymbol{k}^{\prime}$ for $\boldsymbol{k}^{\prime}>\mathbf{0}$. Using the Horikawa surfaces $H\left(8 k^{\prime}-1\right)$ and $H(7) \#_{T} \# H\left(8 k^{\prime}-1\right)$ in Lemmas 2.3 and 2.4 yields the following proposition.

Proposition 3.3. Let $k^{\prime}$ and $n$ be positive integers, $p$ and $q$ be integers greater than 2 , and $G$ be $\{1\}, \mathbb{Z}_{p}$, or $\mathbb{Z}_{p} \oplus \mathbb{Z}_{q}$ (if $n \geq 2$ ) or $\mathbb{Z}, \mathbb{Z} \oplus \mathbb{Z}_{p}$ or $\mathbb{Z} \oplus \mathbb{Z}$ (if $n \geq 1$ ). Then there exist spin irreducible symplectic manifolds $X$ with fundamental group $G$ such that $\left(c_{1}^{2}(X), \chi_{h}(X)\right)$ is either

$$
\left(16 k^{\prime}+8 n-16,8 k^{\prime}+n-2\right)
$$

or

$$
\left(16 k^{\prime}+8 n+88,8 k^{\prime}+n+53\right) .
$$

Corollary 3.4. Each of the manifolds

$$
\begin{aligned}
& H\left(8 k^{\prime}-1\right) \#(2 n-2)\left(S^{2} \times S^{2}\right), \\
& H\left(8 k^{\prime}-1\right) \#(2 n-2)\left(S^{2} \times S^{2}\right) \# L_{p}, \\
& H\left(8 k^{\prime}-1\right) \#(2 n-2)\left(S^{2} \times S^{2}\right) \# L_{q, q}, \\
& H\left(8 k^{\prime}-1\right) \#(2 n-1)\left(S^{2} \times S^{2}\right) \#\left(S^{3} \times S^{1}\right),
\end{aligned}
$$

where $p$ is an integer greater than 1 and $q$ is an odd prime number, admits infinitely many exotic irreducible smooth structures. In each case, only one of these exotic manifolds is symplectic. 


\section{Nonnegative signature}

We now study symplectic manifolds whose signature is either zero or positive.

4.1. J. Park's construction. J. Park [21] used the spin complex surface described in Section 2.6 to find manifolds with trivial fundamental group whose Chern invariants $\left(c_{1}^{2}, \chi_{h}\right)$ are all but finitely many points of the set $\left\{(c, \chi) \in \mathbb{Z}^{2} \mid 0 \leq c \leq 8.74 \chi\right\}$. We filled in the points of negative signature above, and we now follow his construction almost verbatim to address the region $\{(c, \chi) \mid 8 \leq c \leq 8.74 \chi\}$. We start by describing Park's argument and main building blocks.

Consider a simply connected spin symplectic four-manifold $Z$ which contains a symplectic torus $T$ in a cusp neighborhood $N$ and symplectic surface $\Sigma_{g}$ of genus $g$ and zero self-intersection, $\Sigma_{g}$ disjoint from $N$. The Chern coordinates $\left(c_{1}^{2}(Z), \chi_{h}(Z)\right)$ of this manifold are $\left(8 g^{2}-16 g+8,2 g^{2}-g+1\right)$. In particular its signature $\sigma(Z)$ is $-8 g^{2}+8 g$. Now take the spin complex surface described in Section 2.6 and build the symplectic sum of $k$ copies of $Y$ and one copy of $Z$ along a surface of genus $g$ :

$$
X:=Y \#_{\Sigma_{g}} \cdots \#_{\Sigma_{g}} Y \#_{\Sigma_{g}} Z \text {. }
$$

Assume the integer $k$ is such that $X$ has positive signature. Then $\pi_{1}(X)=\{1\}$, since all the pieces are simply connected and the meridian of $\Sigma_{g}$ in $Y-\Sigma_{g}$ is trivial, and, by calculation, the Chern numbers $\left(c_{1}^{2}(X), \chi_{h}(Y)\right)$ are

$$
\left(k c_{1}^{2}(Y)+c(Z)+8 k(g-1), k \chi_{h}(Y)+\chi_{h}(Z)+k(g-1)\right) .
$$

Thus, by considering sufficiently large integers $k$ and $x$,

$$
\frac{c_{1}^{2}(X)}{\chi_{h}(X)}=\frac{k c_{1}^{2}(Y)+c(Z)+8 k(g-1)}{k \chi_{h}(Y)+\chi_{h}(Z)+k(g-1)} \approx \frac{c_{1}^{2}(Y)}{\chi_{h}(Y)} \approx \frac{60068 x^{2}}{6857 x^{2}}=8.76009 \ldots
$$

Next, J. Park fixes sufficiently large integers $k$ and $x$ that $c_{1}^{2}(X)>8.76 \chi_{h}(X)$. At this point one should note that $X$ contains a symplectic torus of self-intersection zero lying on the building block $Z$. One can also find such tori in the $Y$ blocks. To finish his argument, Park defines a line $c=f(\chi)$

$$
f(\chi)=\frac{c(X)}{\chi(X)} \cdot\left(\chi-\frac{1}{2} c(X)-6\right)+c(X)
$$

whose slope $c(X) / \chi(X)$ is greater than 8.76 . Finally, he builds the simply connected manifold $W$ as the symplectic sum $X \#_{T^{2}} X \#_{T^{2}} \cdots \#_{T^{2}} X \#_{T^{2}} V$ along tori of $m$ copies of $X$ and one copy of another manifold $V$ that is chosen to be $H\left(8 k^{\prime}-1\right) \#_{T^{2}} E(2 s)$, $H(7) \#_{T^{2}} H\left(8 k^{\prime}-1\right) \#_{T^{2}} E(2 s)$ or one of the simply connected manifolds constructed in Proposition 3.1. Then, by varying $m$, he shows that for every lattice point $(c, \chi)$ in the first quadrant of the geography plane for which $c=f(\chi)$, there exists an irreducible symplectic simply connected spin four-manifold $W$ with $\left(c_{1}^{2}(W), \chi_{h}(W)\right)=(c, \chi)$.

Since $W$ has a torus $T$ of self-intersection zero and of trivial meridian in $W-T$, Lemmas 2.3 and 2.4 imply the following result (Theorem 1.2). 
Proposition 4.1. Let $G$ be $\{1\}, \mathbb{Z}_{p}, \mathbb{Z}_{p} \oplus \mathbb{Z}_{q}, \mathbb{Z}, \mathbb{Z} \oplus \mathbb{Z}_{p}$ or $\mathbb{Z} \oplus \mathbb{Z}$, where $p$ is an integer greater than 1 and $q$ is an odd prime number. For all but finitely many pairs $(c, \chi)$ of positive integers satisfying the condition

$$
8 \chi \leq c \leq 8.76 \chi
$$

there exists a spin irreducible symplectic manifold $X$ satisfying

$$
\pi_{1}(X)=G \quad \text { and } \quad\left(c_{1}^{2}(X), \chi_{h}(X)\right)=(c, \chi) .
$$

Our next result is about the manifolds with negative signature from the previous proposition.

Corollary 4.2. There exists an integer $N$ such that for all $n \geq N$, each of the manifolds

$$
\begin{array}{ll}
(2 n+1)\left(S^{2} \times S^{2}\right), & (2 n+1)\left(S^{2} \times S^{2}\right) \# L_{p}, \\
(2 n+1)\left(S^{2} \times S^{2}\right) \# L_{q, q}, & (2 n+2)\left(S^{2} \times S^{2}\right) \#\left(S^{1} \times S^{3}\right),
\end{array}
$$

where $p$ and $q$ are odd prime numbers, has infinitely many exotic irreducible smooth structures. In each case, only one of the exotic manifolds admits a symplectic structure.

\section{Acknowledgement}

The author warmly thanks Refik İnanç Baykur for suggesting the problem.

\section{References}

[1] A. Akhmedov, S. Baldridge, R. I. Baykur, P. Kirk and B. D. Park, 'Simply connected minimal symplectic four-manifolds with signature less than -1', J. Eur. Math. Soc. (JEMS) 1 (2010), $133-161$.

[2] A. Akhmedov and B. D. Park, 'Exotic smooth structures on small four-manifolds with odd signature', Invent. Math. 181 (2010), 577-603.

[3] D. Auroux, S. K. Donaldson and L. Katzarkov, 'Luttinger surgery along Lagrangian tori and nonisotopy for singular symplectic plane curves', Math. Ann. 326 (2003), 185-203.

[4] S. Baldridge and P. Kirk, 'Constructions of small symplectic four-manifolds using Luttinger surgery', J. Differential Geom. 82 (2009), 317-362.

[5] R. Fintushel, B. D. Park and R. Stern, 'Reverse-engineering small four-manifolds', Algebr. Geom. Topol. 7 (2007), 2103-2116.

[6] R. Fintushel and R. Stern, 'Surgery in cusp neighborhoods and the geography of irreducible fourmanifolds', Invent. Math. 117 (1994), 455-523.

[7] R. Fintushel and R. Stern, 'A fake four-manifold with $\pi_{1}=\mathbb{Z}$ ', Turkish J. Math. 18 (1994), 1-6.

[8] R. Fintushel and R. Stern, 'Knots, links and four-manifolds', Invent. Math. 134 (1998), 363-400.

[9] R. Fintushel and R. Stern, 'Surgery on nullhomologous tori and simply connected four-manifolds with $b_{2}^{+}=1$ ', J. Topol. 1 (2008), 1-15.

[10] M. Freedman, 'The topology of four-dimensional manifolds', J. Differential Geom. 17 (1982), $357-453$.

[11] R. E. Gompf, 'Nuclei of elliptic surfaces', Topology 30 (1991), 479-511.

[12] R. E. Gompf, 'A new construction of symplectic manifolds', Ann. of Math. (2) 142 (1995), 527-595. 
[13] R. E. Gompf and A. I. Stipsicz, 4-Manifolds and Kirby Calculus, Graduate Studies in Mathematics, 20 (American Mathematical Society, Providence, RI, 1999).

[14] I. Hambleton and M. Kreck, 'Cancellation, elliptic surfaces and the topology of certain fourmanifolds', J. reine angew. Math. 444 (1993), 79-100.

[15] I. Hambleton and P. Teichner, 'A non-extended hermitian form over $\mathbb{Z}[\mathbb{Z}]$ ', Manuscripta Math. 94 (1997), 435-442.

[16] M. J. D. Hamilton and D. Kotshick, 'Minimality and irreducibility of symplectic four-manifolds', Int. Math. Res. Not. IMRN 2006 (2006), article ID 35032, 13 pp.

[17] K. M. Luttinger, 'Lagrangian Tori in $\mathbb{R}^{4}$, J. Differential Geom. 42 (1995), 220-228.

[18] J. McCarthy and J. Wolfson, 'Symplectic normal connect sum', Topology 33 (1994), 729-764.

[19] J. Morgan, M. Mrowka and Z. Szabó, 'Product formulas along $T^{3}$ for Seiberg-Witten invariants', Math. Res. Lett. 4 (1997), 915-929.

[20] J. Park, 'The geography of irreducible four-manifolds', Proc. Amer. Math. Soc. 126 (1998), 2493-2503.

[21] J. Park, 'The geography of spin symplectic four-manifolds', Math. Z. 240 (2002), 405-421.

[22] B. D. Park and Z. Szabó, 'The geography problem for irreducible spin four-manifolds', Trans. Amer. Math. Soc. 352 (2000), 3639-3650.

[23] U. Persson, C. Peters and G. Xiao, 'Geography of spin surfaces', Topology 35 (1996), 845-862.

[24] A. Stipsicz, 'A note on the geography of symplectic four-manifolds', Turkish J. Math. 20 (1996), 135-139.

[25] C. H. Taubes, 'The Seiberg-Witten and Gromov invariants', Math. Res. Lett. 2 (1995), $221-238$.

[26] C. H. Taubes, Seiberg Witten and Gromov Invariants for Symplectic Four-manifolds, First International Press Lecture Series, 2 (International Press, Somerville, 2000).

[27] R. Torres, 'Geography and botany of symplectic four-manifolds with cyclic fundamental group', (2009), arXiv:0903.5503.

[28] R. Torres, 'Irreducible four-manifolds with abelian non-cyclic fundamental group', Topology Appl. 157 (2010), 831-838.

RAFAEL TORRES, Department of Mathematics, California Institute of Technology, 1200 E California Blvd, 91125 Pasadena CA, USA

e-mail: rtorresr@caltech.edu 